\title{
Adenomatoid Tumour of the Epididymis: A Case Report
}

\section{Vishwanath TT, Singh S, Shetty SMCC, Ajay MK}

Department of Radiodiagnosis, JSS Medical College, Mysore, Karnataka, India

\begin{abstract}
Adenomatoid tumours are rare benign neoplasms involving the paratesticular regions, most commonly the tail of epididymis, however they involve various other structures like genitourinary system. Here, we report a case of adenomatoid tumor of the epididymis its ultrasound imaging features and review of literature.
\end{abstract}

Key words: Adenomatoid, Mesothilial, Ultrasound

\section{Introduction}

Adenomatoid tumours of epididymis (ATE) were first described by Golden et al as small mass in scrotal region without any clinical symptoms. These tumors are uncommon and only have an incidence of 5\% and among these about $80 \%$ are known to be benign. Various neoplasms originating from mesenchymal elements may present on the paratesticular tissues. Most of these tumors are smaller in size with mean diameter being less than $2 \mathrm{cms}$ with preponderance to the epididymal tail. Usually ultrasound combined with histopathological examination is diagnostic and conclusive for paratesticular tumours. Surgical excision is helpful and as it usually does not contain a capsule epididymectomy may be necessary.

Correspondence to: Dr. Simran Singh MBBS, Resident, Department of Radiodiagnosis, JSS Medical College, Mysore, Karnataka, India-570004

Email: - dr.simrans@gmail.com

\section{Case Report}

A 40 year old male patient came with complaints of right scrotal lump progressively increasing since 6 months. On clinical examination lump was located on the lower pole of testis but was felt separate from the right testis, with no tenderness and signs of inflammation on inspection. The scrotum wall appeared relatively normal. No history of any trauma was reported.

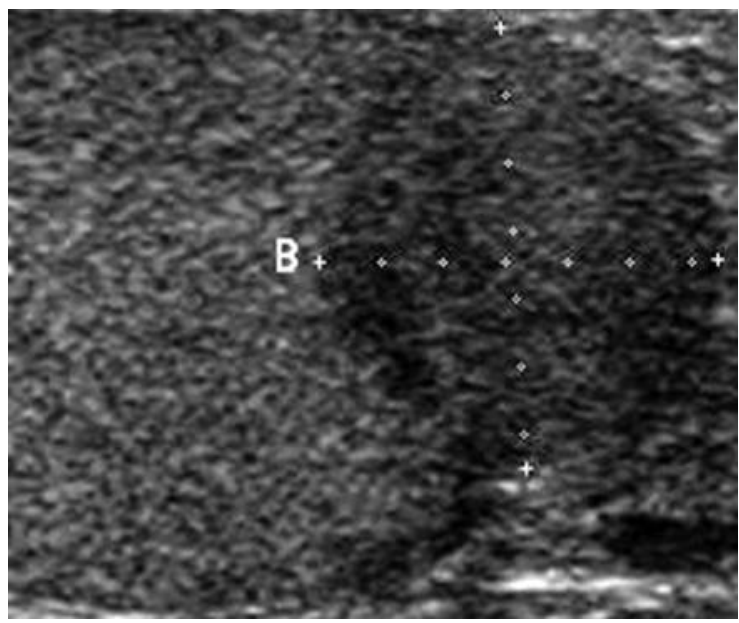

Fig. 1: Grey scale imaging of the mass without any obvious echogenic capsule in the region of tail of right epididymis 
Ultrasound was suggested to rule out epididymorchitis or any malignant mass, ultrasound revealed a mass lesion which was comparatively hypoechoic to testis and without any obvious echogenic capsule in the region of tail of right epididymis. Mass measured $2.6 \times 2.2 \mathrm{cms}$ in size (Fig. 1) and on colour doppler there was internal vascularity was demonstrated. FNAC was suggested by the urologists but it was inconclusive. Patient was taken for surgical exploration and excisional biopsy was done and specimen was sent for histopathological examination which was conclusive of adenomatoid tumor arising from the tail of epididymis on right side (Fig. 2).

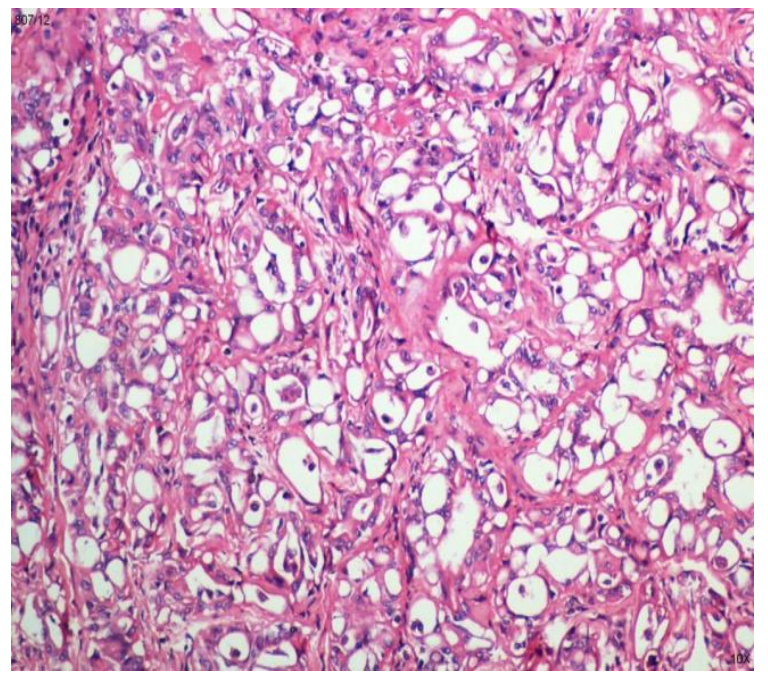

Fig. 2: On histopathology tubules and cords of tumour cells with large intracytoplasmic vacuoles.

\section{Discussion}

Epididymal tumors whether benign or malignant are very rare contributing to only less than $5 \%$ of all the intrascrotal tumors, they are usually of two types primary and secondary and among these epithelial tumors of epididymis are a rare subtype mainly composing of adenomatoid, papillary cystadenoma and leiomyomas. ${ }^{1,2}$

Adenomatoid tumour usually involve the genitourinary system in both males and females, with fallopian tubes, ovaries and uterus being common areas for females and epididymis, testes, spermatic cord, ejaculatory ducts in males (decreasing order of involvement). The main cause for involvement of genital regions is unknown but it may also involve adrenals, lymph nodes, pancreas, mediastinum and pleura. ${ }^{5-8}$

These tumours are usually located in the lower pole of the epididymis (tail) usually as a separate mass with a mean age of presentation between 30 to 40 years, however these tumors can affect any age and cases have been reported in children as well as geriatric age group. Though it can be bilateral but has propensity towards left side than the right side. ${ }^{9}$ These tumors are usually small in size with average size being $2 \mathrm{cms}$ only in diameter but few can also grow up to $10 \mathrm{cms}$ size.

After epididymis the most common location is intratesticular arising from its covering and more common on the lower pole usually seen peripherally and well circumscribed on ultrasonography. Ultrasonography is the initial investigation of choice with high sensitivity and specificity rates. MRI helps in diagnosis as the lesion appears hypointense to surrounding parenchyma arising from testicular surface and enhances less than the normal testis on post contrast administration. ${ }^{14}$

Clinically presentation can vary from being asymptomatic small masses to very painful masses in the scrotum region which can be 
confused for torsion in few, the pain in the testis is usually attributed to the inflammation of the epididymis (epididymitis) but most of these small masses are painless and are incidental findings during routine health check. ${ }^{3,4,10}$

Histogenesis of ATE is not clear where few pathologists consider it to be a reaction to injury or inflammation, most have believed it to be of mesothelial origin. ${ }^{11}$ On electron microscopy, it can present in a wide spectrum however basic patterns being three only i.e. tubules, cell nests and cords. Prominent microvilli, desmosomes and tonofilaments are seen highly suggestive of ATE. ${ }^{12}$ Mesothelial markers like calretinin and HMBE1 may distinguish it from tumors of non mesothelial origin and thus aid in diagnosis.

On histopathology, in our case the tumour was not encapsulated showing cuboidal to flattened tumour cells in cords and tubules having large intracytoplasmic vacuoles which is almost characteristic of adenomatoid tumor.

Ultrasound, being noninvasive, safe, easily available and having high sensitivity and specificity can be used to diagnose benign solid mass lesion of epididymis that are separate from testis. Ultrasound examination demonstrates well circumscribed uniformly hypoechoic mass, that are usually avascular but may demonstrate internal vascularity on colour flow Doppler imaging. Differential diagnosis for epididymis tail solid mass includes fibrous pseudotumour of epididymis, leiomyoma, atrophied extra testis, papillary cystadenomas and angiolipoma. CT and MRI may also help in differential diagnosis on MRI it shows hypointense signal on $\mathrm{T} 1$ with hypointense halo and a hyperintense inner core. ${ }^{13}$

Most surgeons prefer surgical exploration of the epididymis in these cases than needle biopsy as it may lead to easy dissemination in cases of malignancy. FNAC is usually performed but may be inconclusive as was in our case.

A few other paratesticular tumors have been reported like leiomyomas which have spindle cell as characteristic HPE finding. Papillary cystadenomas may be seen in cases of Von Hippel Lindau disease which may show dilated tubules and cysts as characteristic finding in microscopy. A few other differentials can be hemangiomas, chronic epididymitis, spermatocele and neuroectodeermic tumors. Primary malignant tumors of epididymis are only $25 \%$ and are divided into three groups: sarcomas, dysembryonic tumors and epithelial tumors. Treatment is usually surgical followed by radiotherapy and chemotherapy but most of the cases have a poor prognosis.

\section{Conclusion}

Since adenomatoid tumour is a rare benign epididymal tumour, its separation from a more serious testicular tumour is important for surgical management. Ultrasound examination is useful and easy in separating epididymal tumor from testicular tumour. However, MRI maybe also useful to characterise the lesion.

\section{References}

1. Moyano CJL, Giraldez PJ, Sanchez de la Vega J, et al. Adenomatoid tumour of the 
epididymis. Actas Urol Esp 2007; 31: 417.

2. Samada A A, Pereiroa B, Badiola A, Gallegoc C, Zungria E. Adenomatoid tumour of intratesticular localization. Eur Urol 1996; 30: 127-28.

3. Syed Ali A, Tawfeeq AS, Syed Z HJ, Farnaz H, James S AN. Multimodality imaging of paratesticular neoplasms and their rare mimics. Radio Graphics 2003; 23: 1461-76.

4. Tato RJ, Lema GJ, Cimadevila GA, et al. New report of epididymal Adenomatoid tumour. Infrequent pathology. Acta Urol Esp 1997; 21(5): 502-07.

5. Chen KT, Schiff JT. Adenomatoid prostatic tumour. Urology 1983; 21: 8889.

6. Fan K, Johnson DF. Adenomatoid tumour of ejaculatory duct. Urology 1985; 25: 653-54.

7. Evans CP, Vaccaro JA, Storrs BG, Christ PJ. Suprarenal occurrence of an adenomatoid tumour. J Urol 1988; 139: 348-49.
8. Arcadi JA. Adenomatoid tumour in the tunica albuginea of the testis. J Surg Oncol 1988; 37: 38-39.

9. Srigley JR, Hartwick RWJ. Tumours and cysts of the paratesticular region. Pathol Annu 1990; 25: 51-108.

10. Paula JW, Cornelia MS, Isabell A. Extratesticular Scrotal masses. Radiologic-Pathologic Correlation. Radiographics 2003; 23: 215-40.

11. Jackson JR. The histeogenesis of the adenomatoid tumour of the genital tract. Cancer 1958; 11: 337-50.

12. Davy CL, Tang CK. Are all adenomatoid tumours adenomatoid mesothelioma. Hum Path 1981; 12(4): 360-69.

13. Serrano Frago P, Medrano Llorente P, Borque Fernando A, Pascual Llorente M, Allue López M. Consults by scrotum mass: epididymo lesions]. Actas Urol Esp. 2007; 31(4):420-422.

14. Patel MD, Silva AC. MRI of an adenomatoid tumor of the tunica albuginea. AJR Am J Roentgenol. 2004;182(2):415-417. 